\title{
Validação de conteúdo e desenvolvimento de um software para hemodiálise
}

\author{
Content validation and development of a software for hemodialysis \\ Validación de contenido y desarrollo de un software para hemodiálisis \\ Simone Soares da Silva ${ }^{1}$ ib https://orcid.org/0000-0001-9293-2048 \\ Walckiria Garcia Romero Sipolatti' ic https://orcid.org/0000-0002-1365-4797 \\ Bruno Henrique Fiorin ${ }^{1}$ io hitps://orcid.org/0000-0002-1629-9233 \\ Leila Massaroni' ic https://orcid.org/0000-0001-7327-887x \\ Andressa Bolsoni Lopes ${ }^{1}$ io https://orcid.org/0000-0003-1244-5667 \\ Mirian Fioresi ${ }^{1}$ id https://orcid.org/0000-0002-8560-4385 \\ Lorena Barros Furieri ${ }^{1}$ io https://orcid.org/0000-0003-3859-2227
}

\section{Como citar:}

Silva SS, Sipolatti WG, Fiorin BH, Massaroni L, Lopes AB, Fioresi M, et al. Validação de conteúdo e desenvolvimento de um software para hemodiálise. Acta Paul Enferm. 2021;34:AAPE02571.

DOI

http://dx.doi.org/10.37689/actaape/2021A002571

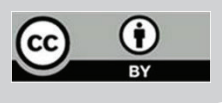

Descritores

Informática em enfermagem; Sistemas de informação; Software; Validação de programas de computador; Enfermagem

Keywords

Nursing informatics; Information systems Software: Computer program validation; Nursing

Decriptores Informática aplicada a la enfermería; Sistemas de información; Software; Validación de programas de computación; Enfermería

$$
\begin{array}{r}
\text { Submetido } \\
4 \text { de Setembro de } 2019 \\
\text { Aceito } \\
1 \text { de Junho de } 2020
\end{array}
$$

\section{Autor correspondente}

Simone Soares da Silva E-mail: simone.soaress1@gmail.com

\section{Resumo}

Objetivo: Descrever o desenvolvimento de um software para o gerenciamento de indicadores clínicos e de qualidade no cuidado de enfermagem de pacientes em hemodiálise.

Métodos: Trata-se de um estudo metodológico, envolvendo três etapas: 1) levantamento do referencial teórico para estruturação de conteúdo acerca de indicadores clínicos e de qualidade, relevantes para registro no serviço de hemodiálise e avaliação da efetividade dialítica; 2) validação de conteúdo por juízes e 3) desenvolvimento do software. Utilizou-se para sua criação a metodologia proposta por Pressman, que consiste em cinco passos: comunicação, planejamento, modelagem, construção e implantação.

Resultados: 0 software produzido compõe-se de 112 itens validados, tendo como funcionalidades 0 sistema de cadastramento e busca de pacientes do serviço, a atualização de dados clínicos e laboratoriais, bem como a geração de relatórios relacionados às infecções, ao implante de acesso vascular, aos eventos adversos, à hospitalização e aos indicadores de qualidade.

Conclusão: Este estudo possibilitou a elaboração de um software como ferramenta para compilação e organização de dados de paciente em terapia hemodialítica, com vista à geração de informações e conhecimentos que subsidie a avaliação e tomada de decisão clínica de enfermagem apoiada em julgamento crítico.

\section{Abstract}

Objective: To describe the development of a software for the management of clinical and quality indicators in patient care under hemodialysis.

Methods: This is a methodological study, which involved three stages: 1) survey of the theoretical framework for structuring content about clinical and quality indicators, relevant for registry in hemodialysis service and assessment of hemodialysis effectiveness; 2) content validation by judges; 3) software development. The methodology proposed by Pressman was used for its creation, which consists of five steps: communication, planning, modeling, construction, and implementation.

Results: The software produced consists of 112 validated items, with the functionality of the system for registering and searching patients at the service, updating clinical and laboratory data as well as generating reports related to infections, vascular access implantation, adverse events, hospitalization and quality indicators.

Conclusion: This study enabled the development of a software as a tool for compiling and organizing patient data on hemodialysis, with a view to generating information and knowledge that supports assessment and clinical decision making of nursing supported by critical judgment. 


\section{Resumen}

Objetivo: Describir el desarrollo de un software para la gestión de indicadores clínicos y de calidad para los cuidados de enfermería de pacientes en hemodiálisis.

Métodos: Se trata de un estudio metodológico, que incluye tres etapas: 1) análisis del marco referencial teórico para la estructuración del contenido sobre indicadores clínicos y de calidad relevantes para el registro en el servicio de hemodiálisis y evaluación de la efectividad didáctica; 2) validación del contenido por jueces, y 3) desarrollo del software. Para su creación, se utilizó la metodología propuesta por Pressman, que consiste en cinco pasos: comunicación, planificación, modelado, construcción y despliegue.

Resultados: El software producido se compone de 112 ítems validados y tiene como funcionalidades el sistema de registro y búsqueda de pacientes del servicio, la actualización de datos clínicos y de laboratorio, así como la generación de informes relacionados con las infecciones, el acceso vascular, los eventos adversos, la hospitalización y los indicadores de calidad.

Conclusión: El estudio permitió la elaboración de un software como herramienta para compilar y organizar datos de pacientes en terapia de hemodiálisis, con el fin de producir información y conocimientos que respalden la evaluación y toma de decisiones clínicas de enfermería basadas en apreciaciones críticas.

\section{Introdução}

A área da saúde tem sido fortemente influenciada pelos avanços tecnológicos que se inserem no cotidiano das instituiçóes de saúde, permeando os processos de trabalho na produção e na incorporação de um corpo de conhecimento necessário à assistência, educação e gestão em saúde. ${ }^{(1,2)}$ No contexto da enfermagem, as tecnologias em saúde se aplicam à organização do serviço, do cuidado e à gestão de pessoas nos diferentes espaços de atuação profissional. ${ }^{(3)}$

O trabalho do enfermeiro se fundamenta na tomada de decisóes clínicas e gerenciais embasadas em evidências científicas que, quando aliada aos recursos da Tecnologia de Informação (TI), tornam o cuidado profissional mais seguro e eficaz. Ainda, possibilita o registro dos dados do paciente com maior segurança, aprimora a comunicação entre os profissionais da equipe e facilita o acesso à informaçáo. ${ }^{(4)}$

No que concerne aos serviços de saúde que prestam assistência a pessoa com doença renal crônica (DRC), é exigido, pela Portaria no 389 GM/MS, de 13 de março de 2014, produzir informações para atender aos indicadores de qualidade definidos e o cumprimento das metas destinadas a garantir o atendimento integral às necessidades de saúde desses pacientes. ${ }^{(5)} \mathrm{E}$ importante destacar que a DRC possui alta incidência, estando presente em 10\% da população mundial e, no Brasil, mais de $120 \mathrm{mil}$ pessoas são pacientes renais crônicos dialíticos, dos quais $93,1 \%$ utilizam a hemodiálise como terapia renal substitutiva. ${ }^{(6)}$

As ferramentas de TI qualificam os processos de trabalho dos profissionais de saúde, permitindo uma ampla visibilidade do serviço, agilidade no acesso e repasse de informaçóes. ${ }^{(7)}$ A utilização de softwares assistenciais de enfermagem, no planejamento do cuidado, viabiliza o registro e armazenamento rigoroso dos dados e, consequentemente, a obtenção de resultados mais confiáveis, diminuindo as chances de erros e corroborando para o suporte à decisão clínica e à segurança do paciente. ${ }^{(8)}$

Nesse sentido, as açóes de enfermagem devem ser acompanhadas com o intuito de se avaliar seus resultados, identificando as fragilidades e os pontos chaves que nortearão o planejamento da melhoria da qualidade assistencial. A avaliação é um processo contínuo de coleta de dados relevantes sobre as respostas do processo saúde-doença e das intervençóes por meio de indicadores, que constituem instrumentos gerenciais para subsidiar a tomada de decisóes relativa à qualidade e segurança do cuidado prestado. ${ }^{(9)}$

Assim, o objetivo deste estudo é descrever o desenvolvimento de um software para o gerenciamento de indicadores clínicos e de qualidade no cuidado de enfermagem a pacientes em hemodiálise.

\section{Métodos}

Estudo metodológico, aprovado pelo Comitê de Ética e Pesquisa (CEP) do hospital do estudo sob CAAE n. 61987216.3.0000.5071, com desenvolvimento tecnológico dividido em três etapas, sendo: 1 ) levantamento do referencial teórico; 2) validação de conteúdo por juízes e 3) desenvolvimento do software.

$\mathrm{Na}$ primeira etapa realizou-se levantamento do referencial teórico para estruturação de conteúdo 
acerca de indicadores clínicos e de qualidade, relevantes para o registro no serviço de hemodiálise e para a avaliação da efetividade dialítica. Foram adicionados dados regidos pela Portaria no 389 GM/ MS, de 13 de março de 2014, que estabelece os indicadores de qualidade que devem ser monitorados pelos estabelecimentos de saúde que prestam assistência às pessoas com $\mathrm{DRC}^{(5)} \mathrm{e}$ dados adicionais que são registrados no serviço de hemodiálise do hospital do estudo para identificação e monitoramento das complicaçóes decorrentes do tratamento dialítico.

$\mathrm{Na}$ segunda etapa, foi efetuada a validação dos conteúdos construídos por juízes. Foram selecionados enfermeiros que atuam no serviço de nefrologia do hospital do estudo há pelo menos dois anos, incluindo hemodiálise de pacientes crônicos e agudos, diálise peritoneal e especialistas em nefrologia ou cursando a especialização.

O contato com os juízes selecionados ocorreu via carta-convite, enviada por meio eletrônico, acompanhado do Termo de Consentimento Livre e Esclarecido (TCLE) e do instrumento no formato online dos formulários do Google Forms, com as seguintes informações: "Caracterização dos enfermeiros/juízes" e o "Instrumento para validação do conteúdo para compor o software para um serviço de hemodiálise". Foram considerados elegíveis os enfermeiros cujas características correspondiam aos critérios de inclusão, que preencheram devidamente os formulários e que enviaram o TCLE assinado.

Para a validação do instrumento, que ocorreu no mês de fevereiro de 2018, foram selecionados, como juízes, nove enfermeiros do serviço de nefrologia e que atuavam na hemodiálise. $\mathrm{O}$ juiz assinalou a pertinência do conteúdo na seguinte escala: 1 . nada pertinente, 2 . pouco pertinente, 3 . indiferente, 4 . muito pertinente e 5 . muitíssimo pertinente.

O método utilizado para quantificar o grau de concordância entre os especialistas foi o Índice de Validação de Conteúdo (IVC), que mediu a porcentagem de juízes que julgaram os conteúdos construídos como muito e muitíssimo pertinentes, ${ }^{(10)}$ sendo considerados totalmente aplicáveis os itens que alcançaram IVC maior ou igual a $0,80 .{ }^{(11)} \mathrm{Os}$ itens que receberam IVC $\geq 0,70$ e $<0,80$ foram revisados de acordo com as sugestóes dos juízes. Após a devolução do instrumento, os dados foram tabulados por meio do programa Microsoft Excel ${ }^{\circledR} 2013$.

A terceira etapa consistiu na elaboração do software utilizando o Modelo Cascata ou Ciclo de Vida Clássico proposto por Winston Royce em 1970, que tem como finalidade estabelecer a ordem no desenvolvimento de grandes produtos de software, e sugere uma abordagem sequencial e sistemática, na qual os requisitos do software sejam bem compreendidos tanto pelo propositor quanto pela equipe de desenvolvimento. Para isso, seguiu-se os seguintes passos propostos por Pressman, 2016: comunicação, planejamento, modelagem, construção e implantação. ${ }^{(12)}$

$\mathrm{Na}$ etapa de comunicação, iniciou-se o projeto com levantamento de requisitos, definição de objetivos, funcionalidades e escopo, bem como elaboração de cronograma de desenvolvimento do sistema.

Foi então planejado o escopo com a equipe de desenvolvedores para estimar custos e detalhes a serem observados nas fases subsequentes.

$\mathrm{Na}$ modelagem, foi criado o protótipo do software através da aplicação das etapas de projeto definidas durante o planejamento. Com a lista de requisitos, foi aplicada uma metodologia ágil de desenvolvimento de projetos, denominada SCRUM, que, através da divisão de etapas do projeto em períodos semanais contínuos, apresentou feedback constante, sendo possível acompanhar o desenvolvimento em todas suas etapas. ${ }^{(13)}$

Para o desenvolvimento do software, foi realizada a codificação e os testes. A linguagem de programação utilizada foi a $C++$ e a plataforma de interface gráfica foi a $Q t$ Creator. ${ }^{(14)}$ Utiliza o MySQL como sistema de gerenciamento de banco de dados, que emprega a linguagem SQL como interface, ${ }^{(15)}$ sendo hospedado no servidor em nuvem da empresa Hostinger. ${ }^{(16)}$

\section{Resultados}

\section{Caracterização dos juízes}

Nove enfermeiros atenderam aos critérios de inclusão e aceitaram participar do estudo. Todos eram do sexo feminino; mais da metade com idade entre 31-35 anos (56\%) e trabalhavam na nefrologia há 4 anos; $33 \%$ trabalham no atendimento aos pacien- 
tes em diálise peritoneal; e cinco enfermeiros (67\%) prestam assistência a pacientes do programa crônico e agudo de hemodiálise.

A maioria dos juízes (89\%) tinham especialização ou residência em nefrologia concluída. Um juiz, especialista em nefrologia, está com mestrado em andamento, e somente um juiz (11\%) está finalizando o curso de especialização.

\section{Validação de conteúdo}

Dos 112 itens e subitens construídos, 63 (56,2\%) alcançaram o escore de IVC=0,89 e $38(33,9 \%)$ alcançaram o escore de 1,0, ou seja, 90,2 \% obtiveram IVC $\geq 0,80$ e eram pertinentes à temática. Somente $11(9,8 \%)$ alcançaram o escore de 0,78 e foram revisados de acordo com as sugestôes dos juízes (Tabela 1).

Tabela 1. Análise de conteúdo dos itens para registro no serviço de hemodiálise

\begin{tabular}{|c|c|}
\hline Itens para registro no serviço de hemodiálise & IVC \\
\hline 1. Número de pacientes em tratamento conservador durante o mês & 1,00 \\
\hline 2. Valor da hemoglobina de pacientes em tratamento conservador & 1,00 \\
\hline 3. Valor do fósforo de paciente em tratamento conservador & 1,00 \\
\hline 4. Pacientes em tratamento conservador encaminhados para tratamento hemodialítico e com FAV maturada & 0,89 \\
\hline 5. Pacientes em tratamento conservador que abandonaram o tratamento & 0,89 \\
\hline 6. Hospitalização dos pacientes em tratamento conservador por intercorrência clínica & 1,00 \\
\hline 7. Número mensal de pacientes em diálise peritoneal & 0,89 \\
\hline 8. Implante de cateter de tenckhoff & 0,89 \\
\hline 9. Troca de extensão de cateter de diálise peritoneal & 0,89 \\
\hline 10. Infecção de cateter de tenckhoff (sinais/sintomas) & 0,89 \\
\hline 10.1. Infecção de cateter de tenckhoff (retirado o acesso) & 0,89 \\
\hline 10.2. Infecção de cateter de tenckhoff (exames colhidos) & 0,89 \\
\hline 10.3. Infecção de cateter de tenckhoff (antibiótico usado) & 0,89 \\
\hline 10.4. Infecção de cateter de tenckhoff (resultado da cultura de líquido peritoneal/swab) & 0,89 \\
\hline 11. Peritonite em pacientes em DPA e DPAC & 0,89 \\
\hline 12. Hospitalização dos pacientes em diálise peritoneal por intercorrência clínica & 0,89 \\
\hline 13. Valor da hemoglobina em pacientes em diálise peritoneal & 0,89 \\
\hline 14. Valor da albumina em pacientes em diálise peritoneal & 0,89 \\
\hline 15. Valor do fósforo em pacientes em diálise peritoneal & 0,89 \\
\hline 16. Valor do PTH de pacientes em diálise peritoneal & 0,89 \\
\hline 17. Óbito em pacientes em diálise peritoneal & 0,89 \\
\hline 18. Pacientes aptos para o transplante e com mais de 6 meses em diálise peritoneal, inscritos na CNCDO & 0,89 \\
\hline 19. Número de pacientes com doença renal crônica em hemodiálise & 1,00 \\
\hline 20. Número de pacientes com doença renal aguda em hemodiálise & 1,00 \\
\hline 21. Implante de cateter venoso central de curta permanência (local) & 1,00 \\
\hline 21.1. Implante de cateter venoso central de curta permanência (motivo) & 1,00 \\
\hline 21.2. Implante de cateter venoso central de curta permanência (data da retirada) & 1,00 \\
\hline 21.3. Implante de cateter venoso central de curta permanência (tempo de uso) & 0,89 \\
\hline 22. Implante de cateter venoso central de longa permanência (local, motivo, data da retirada, tempo de uso) & 1,00 \\
\hline 22.1. Implante de cateter venoso central de longa permanência (motivo) & 1,00 \\
\hline 22.2. Implante de cateter venoso central de longa permanência (data da retirada) & 0,89 \\
\hline 22.3. Implante de cateter venoso central de longa permanência (tempo de uso) & 0,89 \\
\hline 23. Confecção de FAV (Iocal: braquibasílica; braquiocefálica; femural; radiocefálica; ulnar-basílica;). Confecção de prótese de politetrafluoroetileno (PTFE). & 0,89 \\
\hline 24. Infecção de cateter de curta permanência/Cateter duplo lúmen-CDL (sinais/ sintomas) & 1,00 \\
\hline 24.1. Infecção de cateter venoso central de curta permanência/Cateter duplo lúmen-CDL (retirado o acesso) & 1,00 \\
\hline 24.2. Infecção de cateter venoso central de curta permanência/Cateter duplo lúmen-CDL (exames colhidos) & 1,00 \\
\hline 24.3. Infecção de cateter venoso central de curta permanência/Cateter duplo lúmen-CDL (antibiótico usado) & 1,00 \\
\hline 24.4. Infecção de cateter venoso central de curta permanência/Cateter duplo lúmen-CDL (tempo de uso do antibiótico) & 1,00 \\
\hline 24.5. Infecção de cateter venoso central de curta permanência/Cateter duplo lúmen-CDL (resultado da cultura/ swab) & 1,00 \\
\hline 25. Infecção de cateter venoso central de longa permanência/ Permicath @(sinais/sintomas) & 1,00 \\
\hline 25.1. Infecção de cateter venoso central longa permanência/ Permicath @ (retirado o acesso) & 1,00 \\
\hline 25.2. Infecção de cateter venoso central longa permanência/ Permicath® (exames colhidos) & 1,00 \\
\hline 25.3. Infecção de cateter venoso central longa permanência/ Permicath $®$ (antibiótico usado) & 1,00 \\
\hline 25.4. Infecção de cateter venoso central longa permanência/ Permicath @ (tempo de uso do antibiótico) & 1,00 \\
\hline 25.5. Infecção de cateter venoso central longa permanência/ Permicath $®$ (resultado da cultura/swab) & 1,00 \\
\hline 26. Infecção de FAV e prótese (sinais/ sintomas, exames colhidos, antibiótico usado, resultado da cultura/swab) & 0,89 \\
\hline
\end{tabular}


Continuação.

26.1. Infecção de FAV e prótese (exames colhidos)

26.2. Infecção de FAV e prótese (antibiótico usado)

26.3. Infecção de FAV e prótese (resultado da cultura/swab)

27. Trombose de FAV

28. Trombose de prótese politetrafluoroetileno (PTFE)

29. Perda de FAV

30. Outros tipos de infecção durante o período (infecção de trato urinário)

30.1. Outros tipos de infecção durante o período (infecção intestinal ou foco abdominal)

30.2. Outros tipos de infecção durante o período (sítio cirúrgico)

30.3. Outros tipos de infecção durante o período (infecção de pele: infestação parasitária ou celulite infecciosa)

30.4. Outros tipos de infecção durante o período (infecção respiratória/ pneumonia)

31. Número de pacientes em hemodiálise em uso de cateter venoso central de curta duração no último dia de hemodiálise do mês

32. Número de pacientes em hemodiálise em uso de cateter venoso central de longa permanência no último dia de hemodiálise do mês

33. Número de pacientes em hemodiálise em uso de FAV duração no último dia de hemodiálise do mês

34. Número de pacientes em hemodiálise em uso de prótese de politetrafluoroetileno (PTFE) duração no último dia de hemodiálise do mês

35. Hospitalização dos pacientes em hemodiálise por intercorrência clínica

36. Óbito em pacientes em hemodiálise

37. Soroconversão para Hepatite $\mathrm{C}$ positiva em pacientes submetidos à Hemodiálise

38. Soroconversão para Hepatite B em pacientes submetidos à Hemodiálise

39. Valor de hemoglobina de pacientes em hemodiálise

40. Valor de albumina em pacientes em hemodiálise

41. Valor de fósforo de pacientes em hemodiálise

42. Valor de PTH de pacientes em hemodiálise

43. Valor do Ktv de pacientes em hemodiálise

44. Pacientes aptos para o transplante e com mais de 6 meses em hemodiálise, inscritos na CNCDO

45. Fístula arterio venosa- FAV maturadas

46. Eventos adversos (infecção e sinais de infecção (tremores/febre/calafrios)

46.1. Eventos adversos (rompimento de fibras do dialisador)

46.2. Eventos adversos (coagulação do sistema extracorpóreo)

46.3. Eventos adversos (quebra do sistema extracorpóreo)

46.4. Eventos adversos (defeito do material)

46.5. Eventos adversos (uso de material inadequado)

46.6. Eventos adversos (troca de capilares entre pacientes)

46.7. Eventos adversos (erro em punção de FAV)

46.8. Eventos adversos (hematoma)

46.9. Eventos adversos (ruptura de FAV)

46.10. Eventos adversos (desconexão acidental do cateter venoso central com a linha de sangue)

46.11. Eventos adversos (desconexão acidental da agulha da fístula)

46.12. Eventos adversos (reação ao esterilizante)

46.13. Eventos adversos (reação à transfusão de hemoderivados)

46.14. Eventos adversos (reação a medicamentos)

46.15. Eventos adversos (reação a curativos)

46.16. Eventos adversos (lesão de pele)

46.17. Eventos adversos (relacionados ao tratamento de água/ falha na distribuição)

46.18. Eventos adversos (relacionados à máquina de hemodiálise)

46.19. Eventos adversos (queda do paciente)

46.20. Eventos adversos (perda do acesso durante a hemodiálise)

46.21. Eventos adversos (cateter venoso central não funcionante)

46.22. Eventos adversos (fluxo sanguíneo inadequado do acesso)

46.23. Eventos adversos (implante inadequado do cateter venoso central)

46.24. Eventos adversos (sangramento pelo acesso venoso)

46.25. Eventos adversos (erro de prescrição de diálise)

46.26. Eventos adversos (omissão de cuidado)

47. Motivo de entrada (nefroesclerose hipertensiva; nefropatia diabética; glomerulopatias; doença urológica; doença crônica do enxerto; cardiorrenal; hepatorrenal; paciente em trânsito para cirurgia de paratireodectomia ou por internação para outos motivos; lúpus eritemaso sistêmico; outras causas; troca de terapia; síndrome nefrótica; sepse; doença policística ou causas obstrutivas (mieloma).

48. Saída de paciente (mudança de terapia)

48.1. Saída de paciente (recuperação da função renal)

48.2. Saída de paciente (transferência de clínica)

48.3. Saída de paciente (retorno a clínica de origem)

0,89

0,89

0,89

0,89

0,89

1,00

1,00

0,89

0,89

0,89

0,89

1,00

1,00

1,00

1,00

0,89

0,89

0,89

0,89

1,00

1,00

1,00

1,00

1,00

0,89

0,78

0,78

0,89

0,89

0,78

0,89

0,89

0,89

0,78

0,89

0,89

0,89

0,89

0,89

0,89

0,89

0,89

0,78

0,89

0,89

0,89

0,78

0,89

1,00

0,78

0,78

0,89

0,78

1,00

0,89

0,89

0,89

0,89 


\begin{tabular}{ll} 
Continuação. & \\
\hline Itens para registro no serviço de hemodiálise & IVC \\
\hline 48.4. Saída de paciente (paciente em trânsito) & 078 \\
48.5. Saída de paciente (transplante renal) & 0,89 \\
48.6. Saída de paciente (abandono do tratamento) & 0,89 \\
49. Transfusão sanguínea (hemácias, plaquetas, plasma) & 1,00 \\
50. Uso de sacarato de hidróxido férrico & 0,89 \\
51. Uso de eritropoietina (EP0) & 0,89 \\
52. Intercorrências clínicas (hipotensão, hipertensão, náuseas/ vômito, câimbras hipoglicemia/ hiperglicemia) & 1,00 \\
53. Coleta sanguínea mensal, semestral e anual & 0,78 \\
\hline
\end{tabular}

IVC - Índice de Validação de Conteúdo; DPA - Diálise Peritoneal Automatizada; DPAC - Diálise Peritoneal Ambulatorial Contínua; PTH - paratormônio; PTFE - prótese de politetrafluoroetileno; CNCDO - Central de Notificação, Captação e Distribuição de Órgãos e Tecidos; Ktv - Fórmula para mensurar a qualidade da diálise; FAV - Fístula artério venosa; EPO - Eritropoietina

\section{Desenvolvimento do software}

A tecnologia elaborada recebeu o nome de Sistema de Apoio à Hemodiálise (SAHD). É considerada uma tecnologia gerencial, pois é utilizada no gerenciamento da assistência como mediadora da melhora da qualidade. ${ }^{(17)}$ Para funcionamento adequado é necessário que os computadores possuam conexão com a internet para acesso ao servidor.

$\mathrm{O}$ conjunto de funcionalidades requeridas para o desempenho do software foi: elencar pacientes cadastrados no programa de hemodiálise, acrescentar um novo paciente, visualizar e buscar paciente, e atualizar os dados do paciente, como acesso vascular, reuso do seu dialisador e indicadores clínicos e de qualidade.

As funçōes se apresentam ao usuário por meio de um menu inicial, com duas opçóes: novo paciente e lista dos nomes dos pacientes cadastrados. $\mathrm{Na}$ opção "cadastrar paciente", abre-se uma tela que permite cadastramento de informaçóes, criando um banco de dados para organizaçáo e acessibilidade das informaçóes acerca dos pacientes cadastrados no programa de hemodiálise.

O SAHD é iniciado com uma tela de login como mostrado na figura 1-A. Uma vez logado, o profissional poderá consultar, cadastrar paciente e atualizar dados (Figura 2-B e C).

Ao clicar no botão "infecção de acesso", abre-se uma aba onde é possível escolher: o tipo de acesso infectado, sinais e sintomas de infecção, quais exames foram colhidos, se resultado positivo (quais os microrganismos encontrados), antibiótico utilizado e se houve infecção em outro sítio. $\mathrm{Na}$ aba referente aos eventos adversos, há opção de escolha de cerca de 33 ocorrências adversas durante a hemodiálise, podendo selecionar até quatro tipos diferentes. No
A

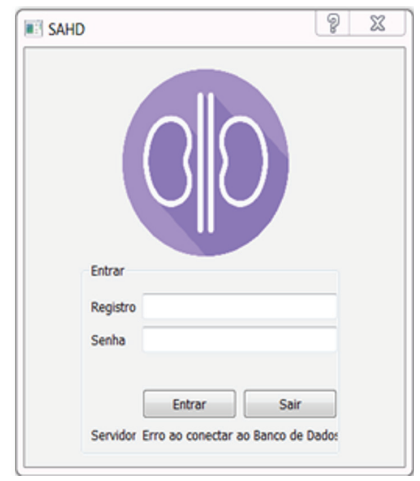

Figura 1. Tela de acesso inicial ao software (A)

B

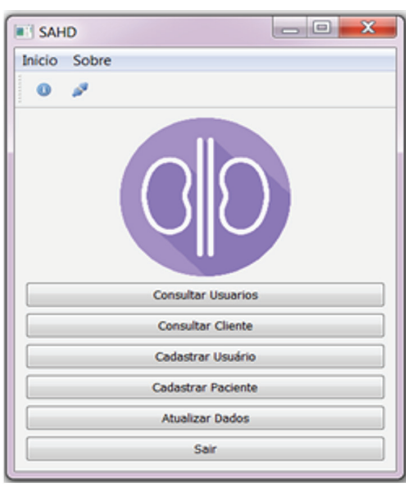

C

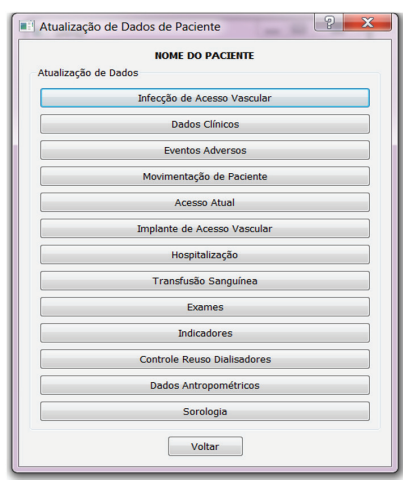

Figura 2. Tela de menu de navegação do software $(\mathrm{B})$ e tela de atualização de dados de paciente cadastrados (C) 
botão "movimentação do paciente", há possibilidade de selecionar qual a situação atual do mesmo: início do tratamento; mudança de terapia renal substitutiva; óbito; evasão; ou outra situação não mencionada. É possível selecionar o atual acesso vascular e isso facilita o monitoramento de implante de novos acessos, de forma que identifique qual tipo implantado, o sítio de inserção mais utilizado e a periodicidade de troca. Hospitalizaçôes, controle de transfusão sanguínea, dados antropométricos e sorologia também podem ser registrados e detalhados. O programa permite a geração de gráficos e tabelas, relacionados à infecção e ao implante de acesso vascular, eventos adversos, hospitalizaçáo e indicadores de qualidade. O software foi registrado no Instituto Nacional da Propriedade Industrial (INPI) via Instituto de Inovação Tecnológica (INIT) da Universidade Federal do Espírito Santo, sob o número BR512019000264-4.

\section{Discussão}

Uma solução bastante aplicável para área da saúde é a construção de softwares e aplicativos, pois são capazes de proporcionar resolutividade, rapidez e segurança, tanto no armazenamento dos dados quanto na apreciação do cliente. ${ }^{(18)} \mathrm{O}$ desenvolvimento das tecnologias impóe que os sistemas de saúde aprimorem a qualidade e que os custos sejam minimizados. Nesse sentido, entram em cena as tecnologias da informação que são capazes de integrar as informaçôes de forma que os profissionais colaborem no cumprimento de metas e na melhoria da qualidade da assistência prestada. ${ }^{(19)}$

$\mathrm{O}$ acesso rápido e facilitado a dados dos pacientes com DRC permite organização e avaliação do serviço, com prestação de assistência segura e de qualidade. Visto que, em muitos serviços de hemodiálise, os dados clínicos e alguns indicadores de qualidade definidos em portaria são coletados, porém, são pouco acessados e discutidos.

Diante desse cenário, o desafio foi desenvolver um software que corresponda às necessidades específicas do setor de hemodiálise e atenda à expectativa de reduzir o tempo despendido na busca de infor- mações por meio de um registro manual, minimizando os erros cometidos. O desenvolvimento do software SAHD é uma ferramenta imperativa no monitoramento do paciente em tratamento hemodialítico, auxiliando a coleta, o armazenamento e a rápida busca de informaçóes. Permite dinamismo na adoção de medidas de intervenção adequadas às intercorrências e eventos adversos, muito comuns durante essa terapia renal substitutiva. ${ }^{(3)}$

A construção do conteúdo por meio de revisão de literatura e posterior validação por enfermeiros foram etapas essenciais para o desenvolvimento do software, visto que o mesmo foi construído baseado em evidências científicas e práticas, e envolveu os enfermeiros, que são o público alvo para utilização da tecnologia. $\mathrm{Ou}$ seja, configura o potencial da interface participativa e colaborativa nas pesquisas metodológicas de produção de tecnologias no campo da enfermagem. ${ }^{(20)}$ Assim, pode contribuir para reduzir a resistência dos profissionais de saúde em utilizar as tecnologias, seja por falta de intimidade com a informática ou por relutância à adesão a novas metodologias de trabalho. ${ }^{(21)}$

O software foi desenvolvido com o intuito de ser incorporado à rotina de trabalho dos profissionais da equipe de saúde do serviço de hemodiálise do hospital do estudo, como ferramenta para o enfrentamento dos desafios daqueles que assistem o paciente portador de doença renal crônica. Para a enfermagem, o programa trará como vantagem a organização do trabalho e permitirá o agrupamento de dados que servirão de subsídios para o cuidado organizado pelo Processo de Enfermagem, ${ }^{(18)}$ que é uma atividade privativa do enfermeiro na qual as necessidades do paciente são identificadas e as metas e cuidados são determinados. ${ }^{(22)}$

Algumas limitaçôes precisam ser apontadas, principalmente pelo software não funcionar interligado ao sistema de prontuários eletrônicos do hospital do estudo. Como meta, pretende-se estabelecer uma interface de funcionamento entre o software desenvolvido e o sistema de prontuários do hospital, além de avaliar o impacto da utilização do SAHD nos indicadores de qualidade e indicadores clínicos no setor estudado. 


\section{Conclusão}

Espera-se que essa tecnologia gerencial contribua para a otimizaçáo dos processos de trabalho da enfermagem compilando os dados, com geraçáo de informaçóes e conhecimento para a organizaçáo do serviço, possibilitando a identificação das necessidades do paciente e implementação dos cuidados pré, trans e após a hemodiálise, sempre embasadas em julgamento clínico. As informaçóes disponibilizadas no sistema poderão subsidiar o enfermeiro na definição, execução e registro do Processo de Enfermagem. Ao sistematizar a assistência o enfermeiro pode otimizar seu tempo com atividades gerenciais, disponibilizando tempo para atividades de assistência.

\section{Colaborações}

Silva SS, Sipolatti WGR, Fiorin BH, Massaroni L, Lopes AB, Fioresi M e Furieri LB declaram que contribuíram com a concepção do estudo, análise e interpretação dos dados, redação do artigo, revisão crítica relevante do conteúdo intelectual e aprovação da versão final a ser publicada.

\section{Referências}

1. Moreira AC, Teixeira FE, Araújo TL, Cavalcante TF, Silva MJ, Cruz AT. Desenvolvimento de Software para o cuidado de enfermagem: revisão integrativa. Rev Enferm UFPE on line. 2016; 10 (Supl 6): 4942-50.

2. Pissaia LF, Costa AE, Moreschi C, Rempel C, Carreno I, Granada D. Impacto de tecnologias na implementação da sistematização da assistência de enfermagem hospitalar: uma revisão integrativa. $R$ Epidemiol Control Infec. 2018;8(1):92-100.

3. Carvalho LA, Thofehrn MB, Amestoy SC, Nunes NJ, Fernandes HN. 0 uso de tecnologias no trabalho em enfermagem: revisão Integrativa. J Nutr Health. 2018;8(1):e188104.

4. Jensen R, Guedes ES, Leite MM. Competências em informática essenciais à tomada de decisão no gerenciamento em enfermagem. Rev Esc Enferm USP. 2016;50(1):112-20.

5. Brasil. Ministério da Saúde. Portaria № 389, de 13 de março de 2014. Define os critérios para a organização da linha de cuidado da Pessoa com Doença Renal Crônica (DRC) e institui incentivo financeiro de custeio destinado ao cuidado ambulatorial pré-dialítico. Diário Oficial da União da República Federativa do Brasil; 17 set 2014 [citado 2019 Abr 29]. Disponível em: http://bvsms.saude.gov.br/bvs/saudelegis/ gm/2014/prt0389_13_03_2014.html

6. Thome FS, Sesso RC, Lopes AA, Lugon JR, Martins CT. Inquérito brasileiro de diálise crônica 2017. J Bras Nefrol. 2019; 41(2):208-14.

7. Gimenes FR, Reis RK, da Silva PC, Silva AE, Atila E. Nursing assessment tool for people with liver cirrhosis. Gastroenterol Nurs. 2016;39(4):264-72.

8. Santos T, Passos LP, Silveira DT. Implantação de sistemas informatizados na saúde: uma revisão sistemática. RECIIS. 2017;11(3):1-11.

9. Báo AC, Amestoy SC, Moura GM, Trindade LL. Quality indicators: tools for the management of best practices in Health. Rev Bras Enferm. 2019;72(2):360-6.

10. Cubas MR, Nóbrega MM, organizador. Atenção primária em saúde: diagnóstico, resultado e intervenções de enfermagem. Rio de Janeiro: Elsevier; 2015.

11. Yamada BF, Santos VL. Construção e validação do Índice de Qualidade de Vida de Ferrans \& Powers: versão feridas. Rev Esc Enferm USP. 2009; 43(Spe): 1105-13.

12. Pressman RS. Engenharia de software: uma abordagem profissional. 8a ed. Porto Alegre: AMGH; 2016.

13. Bissi W. SCRUM - Metodologia de Desenvolvimento Ágil. Campo Digital. 2007;2(1):3-6.

14. The Qt Company. The building blocks of Qt. [Internet]. 2019 [cited 2019 Nov 23]. Available from: https://www.qt.io/qt-framework

15. MySQL. Reference manual. What is MySQL? [Internet]. 2019 [cited 2019 Nov 23]. Available from: https://dev.mysql.com/doc/refman/5.7/ en/what-is-mysql.html

16. Hostinger [Internet] 2019 [citado 2019 Nov 23]. Disponível em: https:// www.hostinger.com.br/

17. Nietsche EA, Backes VMS, Colomé CL, Ceratti RN, Ferraz F. Tecnologias educacionais, assistenciais e gerenciais: uma reflexão a partir da concepção dos docentes de enfermagem. Rev Lat Am Enfermagem. 2005; 13(3):344-53.

18. Lima JJ, Vieira LG, Nunes MM. Computerized nursing process: development of a mobile technology for use with neonates. Rev Bras Enferm. 2018;71 (Suppl 3):1273-80.

19. Barra DC, Almeida SR, Dal Sasso GT, Paese F, Rios GC. Metodologia para modelagem e estruturação do processo de enfermagem informatizado em terapia intensiva. Texto Contexto Enferm. 2016;25(3):e2380015.

20. Teixeira E. Desenvolvimento participativo de tecnologias educacionais: lições aprendidas. Rev Enferm UFPI. 2018;7(4):1-3.

21. Pissaia LF, Costa AE, Moreschi C, Rempel C. Tecnologias da informação e comunicação na assistência de enfermagem hospitalar. Rev Epidemiol Controle Infec. 2017; 7(4): 203-7.

22. Boaventura AP, Santos PA, Duran EC. Theoretical and practical knowledge of the nurse on systematization of nursing care and nursing process. Enferm Global. 2017;16(2):182-94. 